\title{
Fast and Robust Analysis of Dynamic Contrast Enhanced MRI Datasets
}

\author{
Olga Kubassova ${ }^{1}$, Mikael Boesen ${ }^{2}$, Roger D. Boyle ${ }^{1}$, Marco A. Cimmino ${ }^{3}$, \\ Karl E. Jensen ${ }^{4}$, Henning Bliddal ${ }^{2}$, and Alexandra Radjenovic ${ }^{5}$ \\ ${ }^{1}$ School of Computing, University of Leeds, UK \\ olga@comp.leeds.ac.uk \\ 2 The Parker Institute Frederiksberg Hospital, Frederiksberg, Denmark \\ mikael.boesen@dadlnet.dkh, Henning.bliddal@frh.regionh.dk \\ ${ }^{3}$ University of Genoa, Genoa, Italy \\ cimmino@unige.it \\ ${ }^{4}$ Rigshospitalet, Department of Radiology, MRI division, Copenhagen, Denmark \\ 5 Academic Unit of Medical Physics, University of Leeds, Leeds General Infirmary, \\ Leeds, UK \\ sasha@medphysics.leeds.ac.uk
}

\begin{abstract}
A fully automated method for quantitative analysis of dynamic contrast-enhanced MRI data acquired with low and high field scanners, using spin echo and gradient echo sequences, depicting various joints is presented. The method incorporates efficient pre-processing techniques and a robust algorithm for quantitative assessment of dynamic signal intensity vs. time curves. It provides differentiated information to the reader regarding areas with the most active perfusion and permits depiction of different disease activity in separate compartments of a joint. Additionally, it provides information on the speed of contrast agent uptake by various tissues. The method delivers objective and easily reproducible results, which have been favourably viewed by a number of medical experts.
\end{abstract}

\section{Introduction}

Rheumatoid arthritis (RA) is an inflammatory disease associated with pathological alteration of microcirculation within the synovial lining of the diarthroidal joints. It is an extremely painful condition that might result in severe disability. More than 1 per cent of the adult population suffer from RA 1 corresponding to several million people in the USA and about 0.5 million in the UK.

Dynamic Contrast Enhanced Magnetic Resonance Imaging (DCE-MRI) is a technique that provides information about tissue vascularity, perfusion and capillary permeability 2,3] and has evolved as an important method for evaluating various diseases of the musculoskeletal system. Temporal changes of DCE-MRI signal intensity during and immediately after a bolus injection of a contrast agent reflect underlying changes in local concentration of the contrast agent, which are proportional to the extent of tissues' inflammation.

N. Ayache, S. Ourselin, A. Maeder (Eds.): MICCAI 2007, Part II, LNCS 4792, pp. 261-269. 2007.

(C) Springer-Verlag Berlin Heidelberg 2007 
Quantitative analysis of DCE-MRI data is needed for disease diagnosis and after treatment progress evaluation. The analysis can be performed using pharmacokinetic 44 or heuristic methods 56]. The later quantify disease progression in terms of heuristic parameters, such as maximum rate of enhancement $(M E)$, initial rate of enhancement $(I R E)$, and time of onset of enhancement $\left(T_{\text {onset }}\right)$. These parameters are extracted by examining individual signal intensity vs. time curves derived from user defined regions of interest (ROI) or on a voxel-by-voxel basis.

Currently, dynamic curves are derived from an approximately $2-3 \mathrm{~mm}^{2}$ ROI positioned in the area of maximal visual enhancement [7. Measurements of IRE and $M E$ contain both spatial and temporal information making the results vulnerable to the size and position of the ROI [8]. Voxel-by-voxel analysis does not depend on ROI position, and therefore objective and suitable for automation; however such methods [9] do not fully utilise available information and cannot cope with the high degree of noise in dynamic frames.

In this paper we propose a method for automated and efficient extraction of heuristics from signal intensity vs. time curves, making their estimation robust to subjective opinion of the operator and noise effects. This method has been tested on a number of DCE-MRI studies acquired by high and low field scanners and has shown robust and efficient performance.

\section{Materials and Methods}

\subsection{Patients}

The following abbreviations will be used: TR - repetition time; TE - echo time; FOV - field of view; GRE - gradient echo sequence; SE - spin echo sequence; TS - total scanning time after contrast; Gd-DTPA - gadolinium diethylene triamine pentacetic acid.

High field MRI: 10 patients with active RA in the metacarpophalangeal joints (MCPJs) were avaluated. Data was acquired in axial direction (Fig. 1,left) with a high field 1.5T MRI scanner (Gyroscan ACS NT, Phillips Medical Systems, Best, The Netherlands), using $T_{1}$ weighted spoiled GRE sequence, TR/TE: $14 / 3.8$; FOV/imaging matrix: $100 \times 200 \mathrm{~mm} / 128 \times 256$, slice thickness $3 \mathrm{~mm}$. Dynamic sequences of 20 GRE images pre-positioned in 6 planes were acquired every 7.1 s., TS $=142 \mathrm{~s}$. Patients were positioned prone, with arm extended in front of the head and a linear circular $11 \mathrm{~cm}$ diameter surface coil placed on the dorsum of the hand.

Low field MRI: 23 patients with active RA, 4 controls, and 1 patient with no RA but occult wrist pain were scanned with $0.2 \mathrm{~T}$ musculoskeletal dedicated extremity scanner (E-scan, Esaote Biomedica, Genoa, Italy) (Fig. 1, mid., right). The patients were examined in supine position with the hand along the side of the body. Following the Gd-DTPA injection $(0.2 \mathrm{mmol} / \mathrm{kg}$ of body weight), the dynamic sequence was performed resulting in 22-30 consecutive fast SE (TR/TE 100/16, FOV/imaging matrix $150 \times 150 / 160 \times 128)$, or GRE 


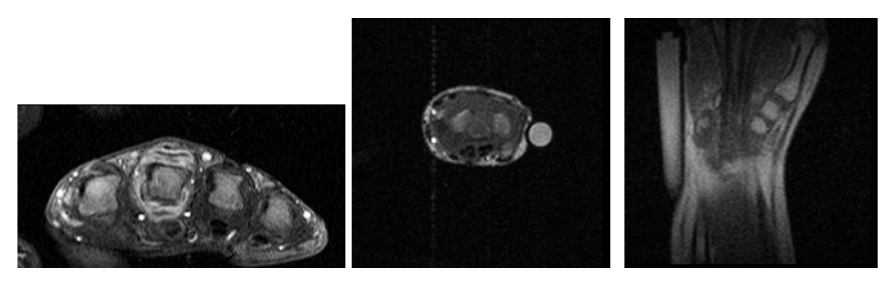

Fig. 1. From the left: post-contrast MR image of MCPJs (high field scanner), postcontrast MR images acquired by low field scanner from the wrist in axial through the first carpal row (SE sequence) and coronal direction through the wrist joint (GRE sequence)

images $(\mathrm{TR} / \mathrm{TE} 60 / 6, \mathrm{FOV} /$ imaging matrix $160 \times 160 \mathrm{~mm} / 256 \times 128)$ in three pre-positioned planes every 10 - 15s., slice thickness $4 \mathrm{~mm}$ in the coronal plane and $5 \mathrm{~mm}$ in the axial plane; TS $=300 \mathrm{~s}$.

\subsection{Quantitative Data Analysis}

The behaviour of signal intensity vs. time curves may be explained by underlying phases of the data acquisition. Starting from a baseline, the perfused tissues absorb the contrast agent, and their intensity climbs up (wash-in phase); it usually increases up to a certain point and then exhibits a plateau (of variable width) followed by wash-out phase (gradual signal intensity decrease).

We propose to use this knowledge of the underlying temporal pattern of the contrast agent uptake to classify and approximate signal intensity vs. time curves normalised over a baseline $(\hat{I})$, as an aid to noise reduction. We imply linear piece-wise approximation; based on the phases of data acquisition observed for DCE-MRI studies involved in RA assessment, we distinguish 4 models $\left(M_{0}-\right.$ $\left.M_{3}\right)$.

$M_{0}$ - negligible enhancement. Some tissues within cortical and trabecular bone, inactive joints, skin and disease unaffected areas do not absorb Gd-DTPA and are not expected to show intensity enhancement in the later frames of temporal slices.

$M_{1}$ - base/wash-in. There is often a proportion of curves in which by the end of the scanning procedure the maximal intensity has not been reached, indicating constant leakage into locally available extra-cellular space. The GdDPTA absorption and signal intensity vs. time curves enhancing continue after the scanning has been completed.

$M_{2}$ - base/wash-in/plateau. Full absorption of the Gd-DTPA by the tissues.

$M_{3}$ - base/wash-in/plateau/wash-out. The wash-out phase is observed at the end of the scanning procedure.

To decide on the 'best' model for a particular voxel, we firstly estimate a noise distribution in the temporal slice (in-slice noise distribution) by approximating 
the first 3 values of $\hat{I}$ (unaffected by any enhancement) and signals corresponding to voxels within the bone interiors by a constant (the local signal mean), with variations being explicit noise measurements. We have illustrated that aggregation of these two noise distributions can be taken as an in-slice noise model [10].

Then, individual $\hat{I}$ are approximated by each of the $M_{0}-M_{3}$ shapes in a least-squares sense; each such 'fit' then implies a number of noise measurements, that are compared using the Kolmogorov-Smirnov statistic to the in-slice noise distribution.

The model in which we have the highest confidence (according to the statistical test) is chosen. Note that we are interested in matching noise distributions and not minimising noise observation; the latter would always preclude the simpler models such as $M_{0}-M_{2}$ in favour of $M_{3} . \hat{I}$ derived from the temporal slices acquired with the low field scanner and corresponding models are shown in Fig. 2,
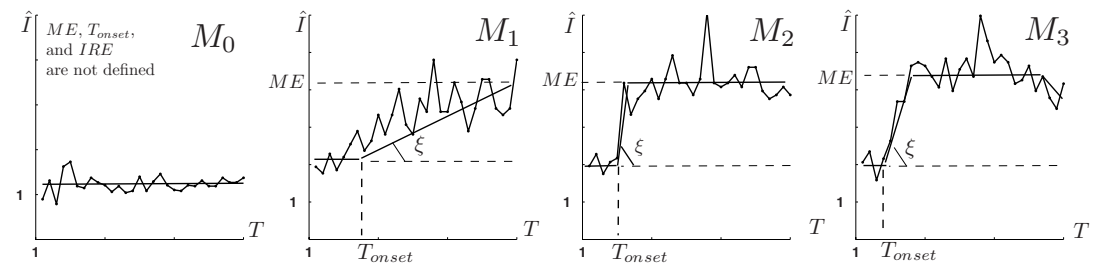

Fig. 2. Estimation of heuristics for each approximation model: $M_{0}-M_{3} . M E$ has not been reached for model $M_{1} . \xi$ is a slope of the $\hat{I}$ that be taken as IRE.

When each voxel is approximated by the 'best' model, we estimate heuristics from the approximations rather than raw data (as shown in Fig. 2) and plot parametric maps (PM) that depict activation events. PMs of $M E$ constructed for temporal slices corresponding to images in Fig. 1 are shown in Fig. 4, each PM is accompanied by the Gd-DTPA uptake map (each voxel is colour-coded according to the model it has assumed: $M_{0}$-no colour, $M_{1}$-red, $M_{2}$-green, $M_{3}$-blue).

Due to the pre-processing techniques applied [11,12] artefactual enhancement has been minimised in PMs constructed with this technique; PMs show preferable characteristics such as sharpness, permitting easier differentiation of structures of interest.

Voxels allocated within blood vessels usually assume $M_{3}$, indicating the presence of the wash-out phase; an affected area is split into several clusters of blue and green, and some areas are coloured red, which allows identification of tissues at which signal intensity did not peak during the acquisition of DCEMRI data. This image allows assessment of the Gd-DTPA kinetics and tissue behaviour classification based on its temporal pattern of the contrast agent uptake. Enhanced voxel count $N_{\text {total }}$ is used as a statistic for quantification of inflammation. 


\section{Results and Discussion}

The algorithm for quantitative analysis of DCE-MRI data has been tested on datasets acquired by high and low field scanners, using SE and GRE sequences, depicting different joints. The disease activity was scored according to the OMERACT RAMRIS [13] evaluation standard for synovitis, bone marrow edema and erosions. Patients processed with low field MRI scanner had a reference fluid in a $10 \mathrm{ml}$ plexiglass, attached along the ulnar long axis of the hand. This was used as a reference for the dynamic calculation.

We have considered 33 patients with active RA (129 temporal slices), 4 healthy controls (12 temporal slices) and 1 patient with no RA (3 temporal slices), but suffering from the wrist pain. Heuristics $M E, I R E$, and pixel count $N_{\text {total }}$ computed with the new technique are significantly higher in the patients with active RA comparing to the controls (Fig. 3). In a temporal slice acquired from the
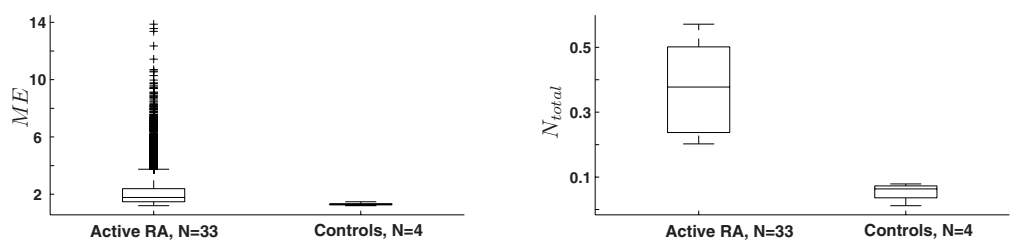

Fig. 3. Box-and-whisker plot of the $M E$ (left) and $N_{\text {total }}$ (right) for patients with active RA and healthy controls $\mathrm{N}$ - is a number of patients
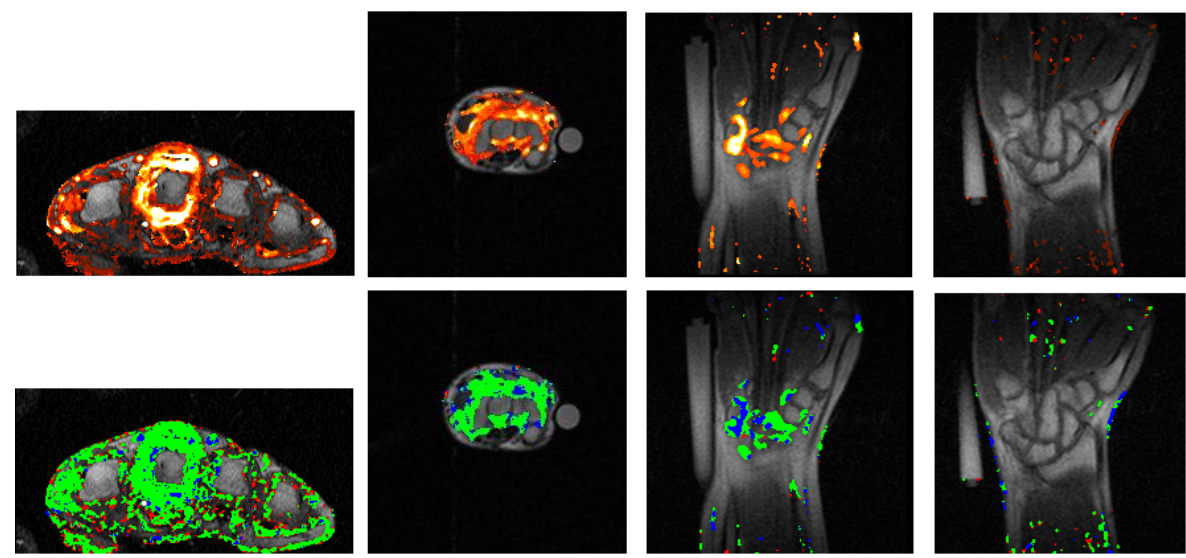

a.

b.

c.

d.

Fig. 4. 1st row: PMs of $M E$ for patients with active RA; data from high field scanner (a.); low field scanner, SE sequence (b); low field scanner, GRE sequence (c.); PM of $M E$ for a healthy control (d.). The colour coding here considers $M E$ and plots lower values in red, moving to yellow then white as the values increase. 2nd row: corresponding Gd-DTPA takeup maps. 

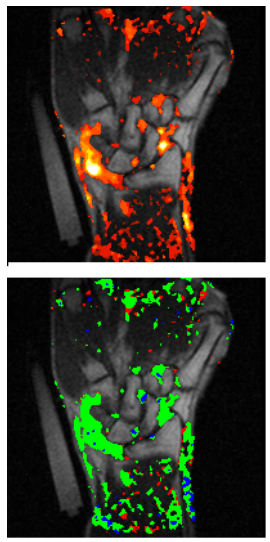
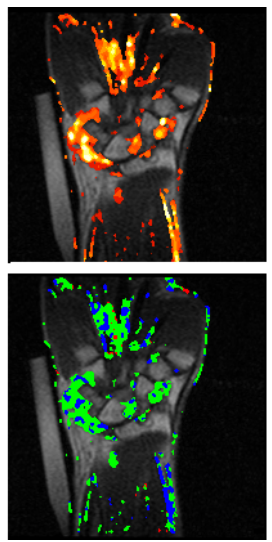
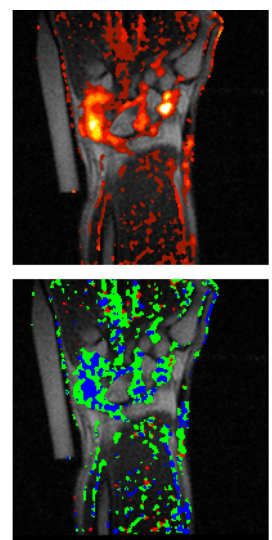

Fig. 5. Parametric map of $M E$ constructed for a patient re-scanned 3 times after injection of the steroid
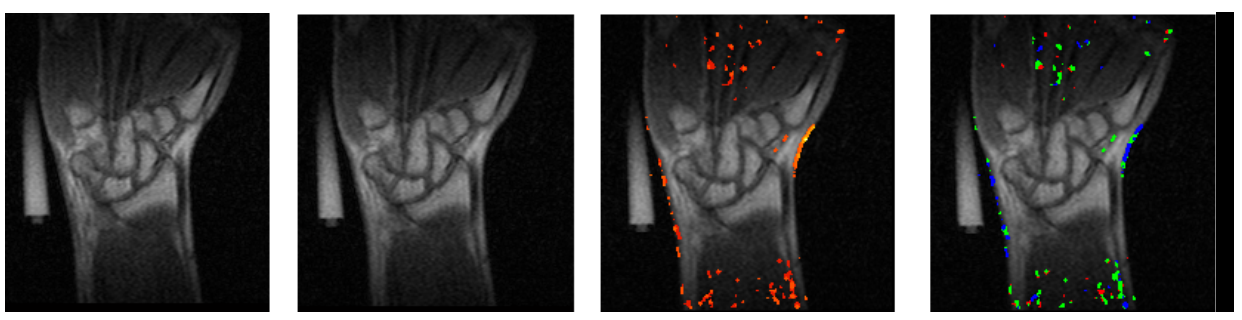

Fig. 6. Pre-, post-contrast images; PM of $M E$ and Gd-DTPA for patient with no RA

patients with active RA by high and low field scanners $M E$ is on average between 2.5 and 4 with maximum reaching 14, and $N_{\text {total }}$ (computed as a ratio between a number of active voxels and the total number of voxels within the joints' exterior) is between 0.3 and 0.4 (depending on the degree of inflammation); for healthy controls $M E$ is on average lower than 1.2 with maximum at 2 and $N_{\text {total }} \in[0,0.05]$. These numbers correlate highly with number of swollen joints.

We have analysed two patients with follow-up scans two weeks after intraarticular glucocorticoid injection; one scanned in the axial plane and the other in the coronal plane. PMs constructed for one of the patients suggest a diminished perfusion in the visible pannus (an expected treatment effect). Such information is not available with conventional $T_{1}$ weighted images after Gd-DTPA contrast or in ultrasound (US) examination, where the patient had high and unchanged MRI synovitis and US colour fraction (inflammation) scores at follow-up.

Another patient had a short clinical relief, but relapsed a few days after each injection with pain and discomfort in the wrist. US as well as static MRI showed no treatment response. This information is reflected by PMs constructed for follow-up examinations (Fig. 5). 
A frequent problem is disease assessment in healthy controls. Usually postand pre-contrast images are compared and ROI is chosen presumptively based on anatomical landmarks. One of the subjects participating in our experiments had no RA, but suffered from occult wrist pain - possibly due to a ganglion in the wrist joint, that we could not find in the post contrast sequences. Pre-, post-contrast images and PM of $M E$ and Gd-DTPA constructed for this subject are shown in Fig. 6.

Visual and quantitative inspection of results obtained with this method allows us to conclude that the patient does not have an inflammatory arthritis, however some tissues exhibit reaction to the contrast agent $\left(N_{\text {total }}=0.06, M E\right.$ is on average 1.3 with maximum at 2.4 ) ; healthy controls normally have less then 0.02 of enhanced pixels; which correspond well to the fact that the ultrasound Doppler showed mild colour Doppler activity in the wrist leading to the conclusion that the patient suffered from a mild unspecific irritation of the wrist.

Our results show that the new method permits depiction of different disease activity in separate compartments of a joint. The method delivers more differentiated information to the reader regarding the areas with most active perfusion. This information is not currently achievable from static $T_{1}$ weighted images, but can to some degree be obtained from the US Doppler images. US on the other hand has the disadvantage of inter observer variations as well as problems with spatial orientation between examinations.

Distribution of the coloured pixels in PMs gives reliable information in the relevant and expected areas of high inflammatory activity. Based on our experiences these areas are in hierarchical order from most to least: the radial and ulnar areas of the proximal part of the wrist, around the ulnar head and to a lesser degree within the inter-carpal and carpo-metacarpal areas. Thus the PMs obtained give the reader information on areas where the patient has most perfusion, and provide more comprehensive information of localised disease activity. This is valuable for disease diagnosis and guidance of intra-articular therapy.

Analysing several corresponding slices, the reader gets a $3 \mathrm{D}$ impression of the inflammatory distribution in the imaged area. It remains to be tested which image plane (axial or coronal) as well as sequence type (SE or GRE) provide the best platform to evaluate the patient's treatment effect in the short and long terms. The proposed method seems to be the most suitable for such judgements.

\section{Conclusion}

Among the randomly chosen patient cohort in this pilot study, PMs are noticeably different, corresponding to our clinical and imaging experiences. Reduction in joints' inflammation has been detected accurately, even when it was not clinically present. Our results demonstrate that the method is sensitive and may be useful in the diagnosis and follow-up examinations of patients who are receiving disease-controlling treatment. 
The method provides a numeric result upon which clinical and research decisions can be confidently made. It is objective and user-independent, which permits generation of easily reproducible and reliable results. Moreover, it allows visual assessment of inflammation and Gd-DTPA uptake previously unavailable.

The possibility of acquisition of the data from low field MRI further extends the usability of the method as low field scanners are more patient friendly and costs are far lower compared to high field scans.

Standardisation of DCE-MRI assessment techniques is crucial and further research will focus on finding correlation between the treatment response seen with different modalities (US, static MRI and dynamic MRI (ROI approach)) and this technique.

\section{References}

1. Silman, A.J., Pearson, J.E.: Epidemiology and genetics of rheumatoid arthritis. Arthritis Research 4(3), S265-S272 (2002)

2. Verstraete, K.L., Deene, Y.D., Roels, H., Dierick, A., Uyttendaele, D., Kunnen, M.: Benign and malignant musculoskeletal lesions: dynamic contrast-enhanced MR imaging-parametric 'first-pass' images depict tissue vascularization and perfusion. Radiology 192(3), 835-843 (1994)

3. Panting, J.R., Gatehouse, P.D., Yang, Z.G., Jerosch-Herold, M., Wilke, N., Firmin, D.N., Pennell, D.J.: Echo-planar magnetic resonance myocardial perfusion imaging: Parametric map analysis and comparison with thallium SPECT. Magnetic Resonance Imaging 13(4), 192-200 (2001)

4. Tofts, P.S.: Modelling tracer kinetics in dynamic Gd-DTPA MR imaging. Journal of Magnetic Resonance Imaging 7(1), 91-101 (1997)

5. d'Arcy, J.A., Collins, D.J., Rowland, I.J., Padhani, A.R., Leach, M.O.: Applications of sliding window reconstruction with Cartesian sampling for dynamic contrast enhanced MRI. NMR in Biomedicine 15(2), 174-183 (2002)

6. Kuhl, C.K., Mielcareck, P., Klaschik, S., Leutner, C., Wardelmann, E., Gieseke, J., Schild, H.H.: Dynamic breast MR imaging: Are signal intensity time course data useful for differential diagnosis of enhancing lesions? Radiology 211(1), 101-110 (1999)

7. Cimmino, M.A., Innocenti, S., Livrone, F., Magnaguagno, F., Silvesti, E., Garlaschi, G.: Dynamic gadolinium-enhanced MRI of the wrist in patients with rheumatoid arthritis. Arthritis and Rheumatism 48(5), 674-680 (2003)

8. McQueen, F.: Comments on the article by Cimmino et al.: Dynamic gadoliniumenhanced MRI of the wrist in patients with rheumatoid arthritis. Arthritis and Rheumatism 50(2), 674-680 (2004)

9. Radjenovic, A.: Measurement of physiological variables by dynamic Gd-DTPA enhanced MRI. PhD thesis, School of Medicine, University of Leeds (2003)

10. Kubassova, O., Boyle, R.D., Radjenovic, A.: Novel method for quantitative evaluation of segmentation outputs for dynamic contrast-enhanced MRI data in RA studies. In: Joint Disease Workshop, Medical Image Computing and Computer Assisted Intervention. vol. 1, pp. 72-79 (2006) 
11. Kubassova, O., Boyle, R.D., Pyatnizkiy, M.: Bone segmentation in metacarpophalangeal MR data. In: 3rd International Conference on Advances in Pattern Recognition, Bath, UK. vol. 2, pp. 726-735 (2005)

12. Periaswamy, S., Farid, H.: Medical image registration with partial data. Medical Image Analysis 10(3), 452-464 (2006)

13. Østergaard, M., Peterfy, C., Conaghan, P., McQueen, F., Bird, P., Ejbjerg, B., Shnier, R., O'Connor, P., Klarlund, M., Emery, P., Genant, H., Lassere, M., Edmonds, J.: OMERACT rheumatoid arthritis magnetic resonance imaging studies. core set of MRI acquisitions, joint pathology definitions, and the OMERACT RAMRI scoring system. Rheumatology 30(6), 1385-1386 (2003) 